\title{
Serum Sarcosine and PSA Levels in Patients With Prostate Cancer in Sokoto, Nigeria.
}

\author{
${ }^{* 1}$ Nnamah,NK, ${ }^{2}$ Anaja, PO, ${ }^{3}$ Mungadi, IA, ${ }^{4}$ Bilbis,LS, and ${ }^{5}$ Dallatu,MK \\ ${ }^{I}$ Department of Chemical Pathology, Faculty of Allied Health Sciences, Nnamdi Azikwe University, Nnewi, \\ ${ }^{2}$ Department of Chemical Pathology, Faculty of Medicine, Ahmadu Bello University, Zaria, \\ ${ }^{3}$ Department of Surgery, Faculty of Clinical Sciences, Usmanu Danfodiyo University, Sokoto, \\ ${ }^{4}$ Department of Biochemistry, Faculty of Science, Usmanu Danfodiyo University, Sokoto, \\ ${ }^{5}$ Department of Chemical Pathology, Faculty of Medical Laboratory Science, Usmanu Danfodiyo University,
} Sokoto, Nigeria

\begin{abstract}
Prostate-specific antigen (PSA) and its derivatives are some of the most commonly used serum biomarker for the detection of prostate disorders in high risk populations.However, there are controversies regarding their clinical usefulness and benefits. In the current work, we aimed to evaluate serum level of sarcosine and these PSA derivatives in 150 prostate cancer $(P C a)$ patients and 200 matched controls in our localities.Results showed serum sarcosine concentration of $64.94 \pm 0.81 \mathrm{nmol} / \mathrm{dl}$ and $134.13 \pm 2.21 \mathrm{nmol} / \mathrm{dl}$, total $P S A$ of $2.75 \pm 0.22 \mathrm{ng} / \mathrm{ml}$ and $33.09 \pm 2.7822 \mathrm{ng} / \mathrm{ml}, f$ PSA of $0.72 \pm 0.0322 \mathrm{ng} / \mathrm{ml}$ and $3.65 \pm 0.21,22 \mathrm{ng} / \mathrm{ml}$ Complexed PSA of $0.72 \pm 0.0322 \mathrm{ng} / \mathrm{ml}$ and $29.54 \pm 2.6622 \mathrm{ng} / \mathrm{ml}$, free PSA of $0.72 \pm 0.0322 \mathrm{ng} / \mathrm{ml}$ and $3.65 \pm 0.2122 \mathrm{ng} / \mathrm{ml}$, percentage free PSA of $2.93 \pm 0.21 \%$ and $14.77 \pm 0.68 \%$, percentage complexed PSA of $70.38 \pm 0.91 \%$ and $85.24 \pm 0.65 \%$, free PSA/complexed PSA ratio of $0.48 \pm 0.02$ and $0.18 \pm 0.01$, free PSA/total PSA ratio of $0.48 \pm 0.23$ and $0.19 \pm 0.11$, complexed PSA/ total PSA ratio of $0.70 \pm 0.01$ and $0.85 \pm 0.0$ in controls and $P C a$ patients respectively. We observed that, group differences in the values of all the analytes studied were signicant $(p<0.05)$, hence we conclude that serum sarcosine and PSA derivatives may be useful markers in the diagnosis of Pca patients in our localities.
\end{abstract}

Keywords: Sereum sarcosine,PSA derivaties, cancer of the prostate.

\section{Introduction}

Prostate cancer is the second leading cause of cancer-related death in men $^{1}$ and several factors present challenging obstacles in developing successful therapeutic modalities and screening tools for its correct detection and treatment ${ }^{2}$. Malignant prostate cells progress through a series of genetic and epigenetic changes leading to aberrant proliferation, angiogenesis, evasion of apoptosis and metastasis to secondry sites ${ }^{3}$ Between 2001 to 2002, the proportion of black patients presenting with high, intermediate and low risk disease in the US, changed to $14.8 \%, 37.5 \%$ and $47.7 \%$ respectively ${ }^{4}$.

As Prostate cancer progression is reported to involve activation of many signaling pathways, intense research efforts to identify serum and tissue biomarkers will expand the opportunities to understand these functional activation of cancer related pathways and consequently lead to correct diagnosis and therapeutic interventions ${ }^{5}$.Just like fingerprint is unique to each person, cancer cells lines exhibit signature proteins pathways, differentiating them from surrounding tissues and other tumors. When these molecular signatures are determined, an individual cancer can be definitely identified, assigned expected pattern of disease progression and therapeutically targeted ${ }^{6}$, hence the rationale for our study.

Sarcosine, an $\mathrm{N}$-methyl derivative of the amino acid glycine, was identified as a differential metabolite that was highly increased during prostate cancer progression to metastasis and can be detected non-invasively in urine. Sarcosine levels were also increased in invasive prostate cancer cell lines relative to benign prostate epithelial cells. Knockdown of glycine- $N$-methyl transferase, the enzyme that generates sarcosine from glycine, attenuated prostate cancer invasion. Addition of exogenous sarcosine or knockdown of the enzyme that leads to sarcosine degradation, sarcosine dehydrogenase, induced an invasive phenotype in benign prostate epithelial cells ${ }^{7}$.Since the introduction of serum prostate-specific antigen (PSA) screening 25 years ago, prostate cancer diagnosis and management have been guided by this biomarker. Yet, PSA has proven controversial as a screening assay owing to several inherent limitations. The next wave multiple prostate cancer biomarkers has emerged, introducing new assays in serum and urine that may supplement or, in time, replace single PSA estimation because of their higher cancer specificity ${ }^{8}$.The development of biomarkers to stratify risk of prostate cancer aggressiveness at the time of screening remains the greatest unmet clinical need in prostate cancer. We reveal here, that serum sarcosine and other PSA derivatives can be potentially important metabolic intermediaries in patients with prostate cancer. 


\section{Materials And Methods}

One hundred and fifty (150) Pca patients aged 30-90 years, from the Urology Unit, Usmanu Danfodiyo University Teaching Hospital Sokoto, Nigeria, who had undergone Transrectal Ultra Sonography (TRUS), Digital Rectal Examination (DRE), and/or histologically confirmed and diagnosed to have either prostate cancer were recruited in the study. Two hundred(200) Control subjects, who were apparently healthy volunteers from among the staff in the hospital and other volunteers were also recruited in the study. Informed consent from all the participant and institutional ethical approval was obtained. Serum sarcosine was measured by the Colourimetric method ${ }^{9}$ ( BioVision Research Products, 980 Linda Vista Avenue, Mountain View, CA 94043 USA. ).Total and free Prostate Specific Antigen (tPSA,fPSA) was estimated by Elisa method ${ }^{10}$ while the remaining PSA derivatives were calculated mathematically.The data were analysed statistically for the diagnostic performance of the parameters in PCa patients using Anova, T-test and ROC curves in SPSS package version 17

\section{Results}

Results of the current work are shown on tables 1 to 3 and figures 1 to 3 . Table 1 shows mean values of serum sarcosine in patients and controls, table 2. mean values of some clinical parameters in patients and controls, while table 3 shows mean values of PSA ratios in patients and controls. Figure 1 shows ROC Curve for total PSA, free PSA and Blood Sarcosine for the prostate cancer group, Figure2 shows ROC Curve for total PSA, Complexed PSA and Blood Sarcosine fo the cancer group in PSA range 0-10ng/ml while Figure 3 shows ROC Curve for total PSA, free PSA and Blood Sarcosine for the Cancer group in PSA range 0-10ng/ml.

Both tables and figures are showing a significant difference $(\mathrm{p}<0.05)$ between the values observed in Pca patients and control subjects in all the analytes studied. These may indicate the usefulness of these biomarkers in prostate cancer state.

\section{Discussion}

Prostate cancer $(\mathrm{PCa})$ is the second most frequently diagnosed cancer and the sixth leading cause of cancer deaths in males, accounting for $14 \%$ of the total new tumor cases and $6 \%$ of the total male cancer deaths in $2008^{1}$. Currently, early detection of PCa relies primarily on an abnormal digital rectal examination (DRE) and an elevated prostate-specific antigen (PSA) level leading to a prostate biopsy. However, because of the low specificity of PSA, up to $75 \%$ of men with PSA levels of $2-10 \mathrm{ng} / \mathrm{ml}$ and/or a suspicious DRE have a negative first biopsy ${ }^{11}$. In order to reduce the number of unnecessary biopsies, many probability-based algorithms have been developed, based on additional relevant prebiopsy information like the use of different molecular forms of PSA such as free and total PSA (fPSA,t PSA) and other PSA derivatives ${ }^{12,13}$.

In the current work, we report for the first time, serum level of sarcosine in patients with prostate cancer in our environment and surprisingly, this biomarker significantly differentiate our Pca patients from controls. Elsewhere, it was reported that, sarcosine was significantly increased during disease progression from benign to PCa to metastatic disease, and was detectable in urine also ${ }^{14}$, reported that when the analysis was restricted to patients with PSA values in the grey zone of $2-10 \mathrm{ng} / \mathrm{ml}$, sarcosine performed better than PSA in differentiating biopsy-positive PCa patients from biopsy negative controls, with an AUC of 0.69 as compared to an AUC of 0.53 for PSA. Regardless of the specimen type, sarcosine was significantly higher in PCa patients than in controls, thus, sarcosine seems to have the potential to identify patients with PCa in a particular cohort of low range PSA subjects ${ }^{14}$

In the current work also, we were able to show for the first time, the diagnostic potentials of some hitherto, not reported PSA derivatives in our laboratories. Serum levels of total, free, complexed, percentage complexed and percentage free PSA are all significantly higher in our Pca patients than in controls.Additionally, calculated values of free to total, free to complexed and complexed to total ratios were significantly higher in the Pca patients than controls. Currently, \% fPSA is approved for use to improve PSA accuracy in men ${ }^{6}$.

There has been a great deal of investigation aimed at improving the accuracy of PSA-based screening. Adjunctive measurements considering the rate of PSA changes with time, the ratios of free and proteinbound PSA, and the relationship of PSA to prostate size have improved performance characteristics in some setting ${ }^{15}$. Several nomograms combining PSA with other clinical variables have also been developed to improve prognostic value beyond that of individual tests ${ }^{16}$

Clinical significance of these PSA derivatives has been reported elsewhere. Serum PSA circulates in either an unbound "free" form or bound to one of several proteins, most frequently alpha-1-antichymotrypsin $(\mathrm{ACT})^{17}$. Levels of free PSA (fPSA) can be detected and compared to total PSA, yielding the proportion of free PSA (\%fPSA). Studies have also shown that men with the highest proportions of complexed PSA are more likely to have prostate cancer ${ }^{18}$ and that \%fPSA is lower in men with prostate cancer as compared to benign prostatic hyperplasia (BPH), hence these derivatives also have differential diagnosis value ${ }^{19}$. Thus, \%fPSA showed promise in distinguishing malignant from benign prostate disease. 
Is worthy to note, proliferating cancer cells exhibit considerably different metabolic requirements to most normal differentiated cells, and In order to support their high rates of proliferation, cancer cells consume additional nutrients and divert those nutrients into other synthesis pathways probably sarcosine, PSA and its derivatives. High-throughput analysis of these metabolites offers a new tool for diagnosis and treatment. Comprehensive analysis of the complete set of these intermediates and metabolic products ${ }^{20}$.can be used to characterize the "metabolic fingerprint" of a tumor as attempted in this study, and identify novel biomarkers that may be potentially useful for early diagnosis and monitoring therapeutic efficacy.

In conclusion, we provide evidence that serum sarcosine and other PSA derivatives, may have additional predictive value to PSA. Even if these results are regarded as preliminary, serum sarcosine in association with other tumor markers, such as PSA derivatives could have an important role in PCa diagnosis and increasing the accuracy of its detection. Series of comprehensive blinded validation studies are warranted to confirm the utility of these biomarkers.

\section{References}

[1]. Jemal A, Bray F, Center MM, Ferlay J, Ward E and Forman D. (2011). Global cancer statistics. CA Cancer J Clin;61(2):69-90.

[2]. Schröder,,FH,, Jonas Hugosson., Monique J. Roobol, Teuvo L.J. Tammela, et al.(2009) Screening and Prostate-Cancer Mortality in a Randomized European Study. N Engl J Med; 360:1320-1328.

[3]. Fornaro M, Plescia J, Chheang S, Tallini G, Zhu YM, King M, Altieri DC and Languino LR (2003) .Fibronectin protects prostate cancer cells from tumor necrosis factor-alpha-induced apoptosis via the AKT/survivin pathway. J Biol Chem. 2003 Dec 12;278(50):50402-11

[4]. Matthew,R. C,Deborah,P. L,Shilpa, S and Mehtapeter R. C. (2003) Time Trends in Clinical Risk Stratification for Prostate Cancer: Implications for Outcomes (Data From CaPSURE). The Journal of Urology, 170 :( 6), S21-S27

[5]. Martin AJ, Lord SJ, Verry HE, Stockler MR, Emery JD (2013)Risk assessment to guide prostate cancer screening decisions: a costeffectiveness analysis. Med J Aust. 3;198(10):546-50.

[6]. Jeffrey T and Stacy L (2010) PSA and Beyond: The Past, Present, and Future of Investigative Biomarkers for Prostate Cancer. The Scientific World JOURNAL, 10: 1919-1931

[7]. Arun S, Laila M. P, Thekkelnaycke M. R. et al,(2009).Metabolomic profiles delineate potential role for sarcosine in prostate cancer progression. Nature 457, 910-914.

[8]. John R. P,Mark A. R, John T. W ${ }^{4}$ and Arul M. C. (2012)Beyond PSA: The Next Generation of Prostate Cancer Biomarkers. Sci.Trans Med. 4:( 127), 127-

[9]. Tietz, N.W. (1995): Testosterone, In: Clinical Guide to Laboratory Test, $3^{\text {rd }}$ Edition. Pp578-580. WB Saunders Coy. .

[10]. Stowell,LH, Sharman,LE and Hamel, K.An enzyme-linked Immunosorbent Assay (ELISA) for prostate specific antigen. Forensic Science Intern.50:125-138,1991.

[11]. Postma R and Schro “der FH (2005). Screening for prostate cancer. Eur J Cancer;41(6):825-833.

[12]. Fiorentino M, Capizzi E and Loda M (2010). Blood and tissue biomarkers in prostate cancer: State of the art. Urol Clin North Am; 37(1):131-141.

[13]. Ploussard G and de la Taille A (2010). Urine biomarkers in prostate cancer. Nat Rev Urol ;7(2):101-109.

[14]. Sreekumar A, Poisson LM, Rajendiran TM, Khan AP, Cao Q, Yu J. et al (2010). Re: Florian Jentzmik, Carsten Stephan, Kurt Miller. Sarcosine in urine after digital rectal examination fails as a marker in prostate cancer detection and identification of aggressive tumours (Eur Urol;58:12-8). Eur Urol ;58(3): e29-30.

[15]. Petrovics G, Liu A, Shaheduzzaman S, Furusato B, Sun C, Chen Y, Nau M, Ravindranath L, Chen Y, Dobi A, Srikantan V, Sesterhenn IA, McLeod DG, Vahey M, Moul JW and Srivastava S (2005). Frequent overexpression of ETS-related gene-1 (ERG1) in prostate cancer transcriptome. Oncogene;24(23):3847-3852.

[16]. Winnes M, Lissbrant E, Damber JE and Stenman G (2007). Molecular genetic analyses of the TMPRSS2-ERG and TMPRSS2ETV1 gene fusions in 50 cases of prostate cancer. Oncol Rep;17(5): 1033-1036.

[17]. Rubio-Briones J, Fernandez-Serra A, Calatrava A, Garci 'aCasado Z, Rubio L, Bonillo MA, Iborra I, Solsona E and Lo pezGuerrero JA (2010). Clinical implications of TMPRSS2-ERG gene fusion expression in patients with prostate cancer treated with radical prostatectomy. J Urol;183(5):2054-2061.

[18]. Welch HG, Schwartz LM and Woloshin S (2005). Prostate-specific antigen levels in the United States: Implications of various definitions for abnormal. J Natl Cancer Inst; 97(15):1132- 1137.

[19]. Auprich M, Bjartell A, Chun FK, de la Taille A, Freedland SJ, Haese A, Schalken J, Stenzl A, Tombal B and van der Poel H (2011). Contemporary role of prostate cancer antigen 3 in the management of prostate cancer. Eur Urol ;60(5):1045-1054.

[20]. Spratlin JL, Serkova NJ and Eckhardt SG (2009). Clinical applications of metabolomics in oncology: A review. Clin Cancer Res ; $15(2): 431-440$.

Table 1. Mean values of serum sarcosine $(m e a n \pm S E M)$ in patients and controls

\begin{tabular}{|l|l|l|}
\hline Subjects & N & Blood sarcosine (nmol/dl) \\
\hline PCa & 150 & $134.13 \pm 2.21$ \\
\hline Controls & 200 & $64.94 \pm 0.81$ \\
\hline F & & $91.905(72.782)$ \\
\hline p-value & & $<0.01$ \\
\hline PCa vs Control & & $<0.05$ \\
\hline
\end{tabular}

$\mathbf{n}=$ sample size

SEM = standard error of mean,

$\mathrm{PCa}=$ Prostate cancer

$\mathrm{P}-$ value $=$ significant 
Serum Sarcosine and PSA Levels In Patients With Prostate Cancer in Sokoto, Nigeria.

Table 2. Mean values of some clinical parameters (mean \pm SEM) in patients and controls

\begin{tabular}{|l|l|l|l|l|l|l|}
\hline Subjects & N & tPSA $(\mathbf{n g} / \mathbf{m l})$ & fPSA $(\mathbf{n g} / \mathbf{m l})$ & CPSA $(\mathbf{n g} / \mathbf{m l})$ & \% fPSA & \% cPSA \\
\hline PCa & 150 & $33.09 \pm 2.78$ & $3.65 \pm 0.21$ & $29.54 \pm 2.66$ & $14.77 \pm 0.68$ & $85.24 \pm 0.65$ \\
\hline Controls & 200 & $2.75 \pm 0.22$ & $0.72 \pm 0.03$ & $0.72 \pm 0.03$ & $2.93 \pm 0.21$ & $70.38 \pm 0.91$ \\
\hline F & & 16.767 & $22.189(16.767)$ & 15.772 & 13.627 & 21.117 \\
& & $(22.340)$ & & $(21.117)$ & $(11.441)$ & $(11.441)$ \\
\hline p-value & & $<0.01$ & $<0.1$ & $<0.01$ & $<0.01$ & $<0.01$ \\
\hline PCa Vs Control & & $<0.05$ & $<0.05$ & $<0.05$ & $<0.05$ & $<0.05$ \\
\hline
\end{tabular}

$\mathbf{n}=$ sample size

SEM = standard error of mean

$\mathbf{P C a}=$ Prostate cancer

tPSA $=$ total prostate specific antigen

fPSA $=$ free prostate specific antigen

CPSA = complexed prostate specific antigen

$\mathbf{P}$ value $=$ Significant

Table 3. Mean values of PSA ratios (mean \pm SEM) in patients and control

\begin{tabular}{|l|l|l|l|l|}
\hline Subjects & $\mathbf{N}$ & fPSA/cPSA & fPSA/tPSA & cPSA/tPSA \\
\hline PCa & 150 & $0.18 \pm 0.01$ & $0.19 \pm 0.11$ & $0.85 \pm 0.01$ \\
\hline Controls & 200 & $0.48 \pm 0.02$ & $0.48 \pm 0.23$ & $0.70 \pm 0.01$ \\
F & & $5.758(6.856)$ & $8.693(7.306)$ & $11.441(13.627)$ \\
\hline p-value PCa vs & & $<0.01$ & $<0.01$ & $<0.01$ \\
$\quad$ Control & & $<0.05$ & $<0.05$ & $<0.05$ \\
\hline
\end{tabular}

$\mathbf{n}=$ sample size

SEM = standard error of mean

$\mathbf{P C a}=$ Prostate cancer

tPSA = total prostate specific antigen

fPSA $=$ free prostate specific antigen

CPSA = complexed prostate specific antigen

$\mathrm{P}$ - value: significant

ROC Curve

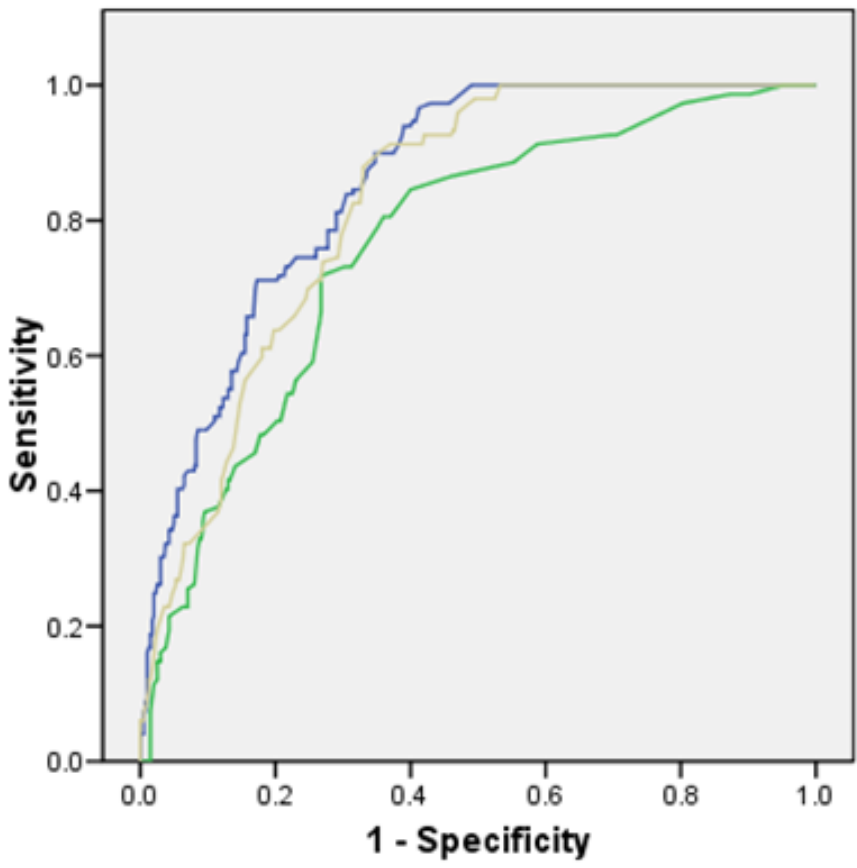

Source of the Curve

- TotalPSA

- FreePSA

BloodSarcosine

Diagonal segments are produced by ties.

Fig 1.ROC Curve for total PSA, free PSA and Blood Sarcosine for the prostate cancer group PSA $=$ Prostate Specific antigen 


\section{ROC Curve}

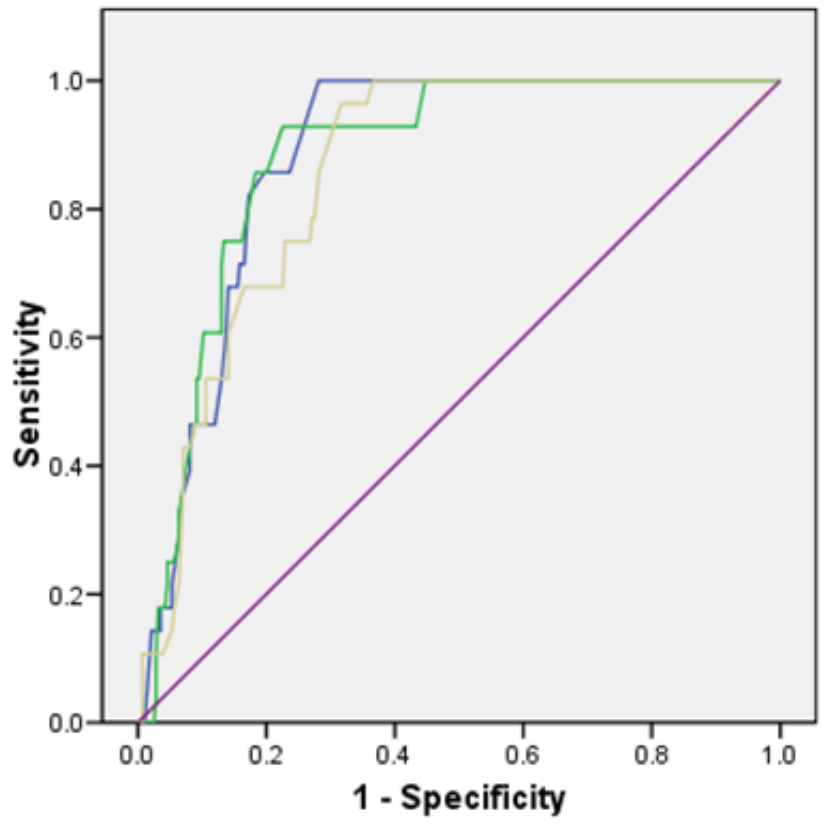

Source of the Curve

- TotalPSA

- compPSA

BloodSarcosine

- Reference Line

Diagonal segments are produced by ties

Fig.2.ROC Curve for total PSA, Complexed PSA and Blood Sarcosine for the cancer group in PSA range $0-10 \mathrm{ng} / \mathrm{ml}$

PSA = Prostate Specific antigen

$\mathbf{P C a}=$ Prostate Cancer

ROC Curve

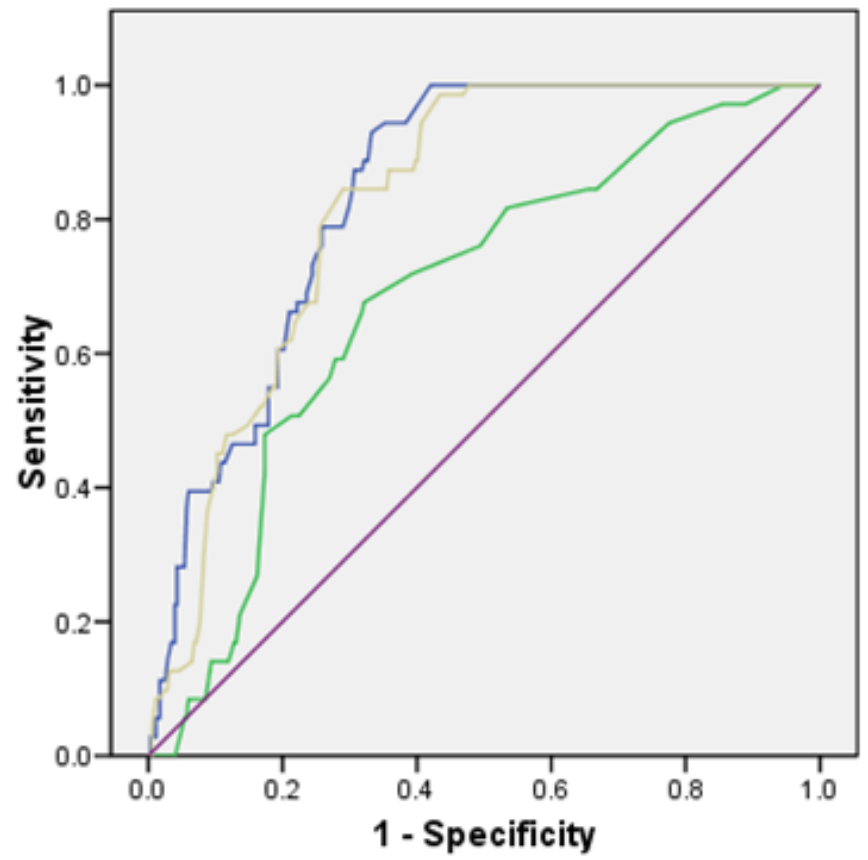

Source of the Curve

- TotalPSA

- FreePSA

BloodSarcosine

- Reference Line

Diagonal segments are produced by ties.

Fig 3.ROC Curve for total PSA, free PSA and Blood Sarcosine for the Cancer group in PSA range $0-10 \mathrm{ng} / \mathrm{ml}$ 